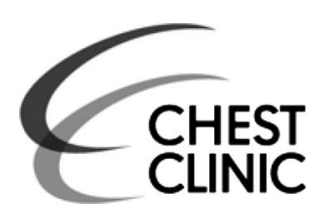

${ }^{1}$ Department of Thoracic Surgery, University of Rome Sapienza, Rome, Italy ${ }^{2}$ Department of Radiology, University of Rome Sapienza, Rome, Italy

\section{Correspondence to}

Dr Marco Anile, Department of Thoracic Surgery, University of Rome Sapienza, Viale del Policlinico 155 00161, Rome, Italy; marco.anile@uniroma1.it

Received 19 January 2015 Revised 6 March 2015 Accepted 11 March 2015 Published Online First 31 March 2015

\title{
Right intralobar sequestration with arterial supply from left gastric artery
}

\author{
Ilaria Onorati, ${ }^{1}$ Daniele Diso, ${ }^{1}$ Michele Anzidei, ${ }^{2}$ Tiziano De Giacomo, ${ }^{1}$ Marco Anile ${ }^{1}$
}

A 61-year-old man with recurrent episodes of haemoptysis and pneumonia underwent chest CT scan showing an intralobar sequestration in the right lower lobe (figure 1); at the multiplane CT reconstruction, the artery supplying the sequestration arose from the left gastric artery (figure 2). The patient underwent right lower lobectomy with closure of the aberrant arterial vessel by mechanical stapler. The postoperative course was uneventful, and the patient was discharged on the fifth postoperative day. The arterial supply arising from left gastric artery is a rare condition; ${ }^{1}$ in our knowledge, this is the first adult ${ }^{2}$ case supplying a right intralobar sequestration.

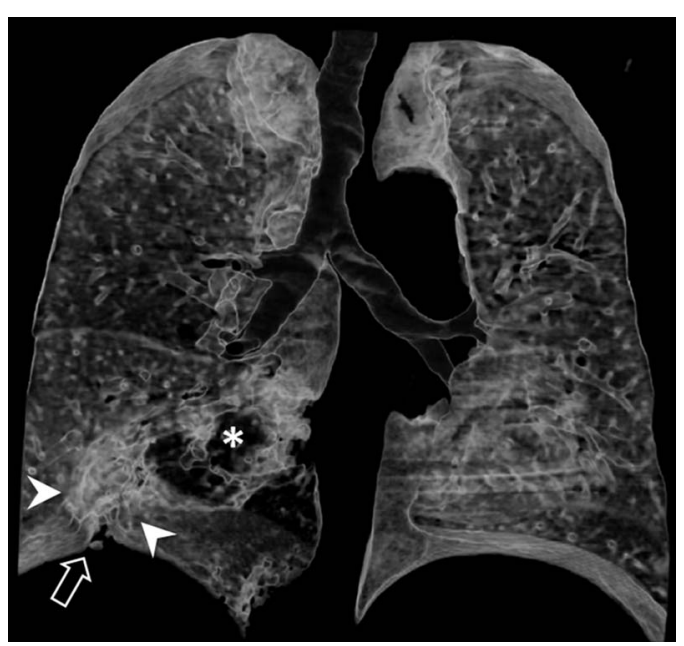

Figure 1 Volume rendering reconstruction (frontal view) from unenhanced $\mathrm{CT}$ acquisition demonstrates an area of increased attenuation in the right lung base (white arrowheads), with retraction of adjacent pleura (open arrow), consistent with intralobar pulmonary sequestration. Image also shows an area of decreased attenuation (asterisk) adjacent to the sequestration, consistent with hyperinflated parenchyma due to segmental bronchial obstruction.

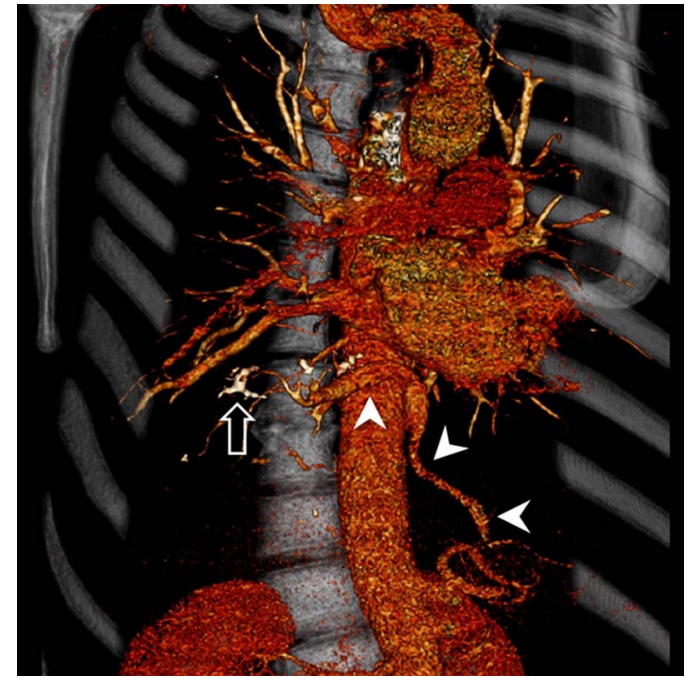

Figure 2 Volume rendering reconstruction (right oblique view) from contrast-enhanced CT acquisition shows an anomalous arterial vessel arising from the left gastric artery (white arrowheads), coursing through the diaphragm and feeding the sequestration in the right lung base. Image also demonstrates an area of very high attenuation (open arrow), consistent with flebolytes.

Competing interests None.

Patient consent Obtained.

Ethics approval Review Board of Department.

Provenance and peer review Not commissioned; internally peer reviewed.

\section{REFERENCES}

1 Juettner FM, Pinter $H H$, Friehs $G B$, et al. Bronchial carcinoid arising in intralobar bronchopulmonary sequestration with vascular supply from the left gastric artery. Case report. I Thorac Cardiovasc Surg 1985;90:25-8.

2 Fukuzawa R, Komori K, Kohno T, et al. Bilateral intralobar sequestration of the lung with a bridging isthmus: pathologic and radiologic findings. Pediatr Dev Pathol 2014;17:55-8. 